\title{
Technè
}

La science au service de l'histoire de l'art et de la préservation des biens culturels

46 | 2018

Science et conservation

\section{Les rayons UV-C comme solution alternative aux traitements chimiques pour l'élimination des micro-organismes chlorophylliens}

Une étude de cas : la Vierge à l'Enfant de Poitiers

The use of UVC rays as an alternative solution to chemical treatments to

eliminate chlorophylian microorganisms: the Poitiers Virgin and Child

\section{Camille Devilliers et Faisl Bousta}

\section{OpenEdition}

Journals

Édition électronique

URL : http://journals.openedition.org/techne/438

DOI : $10.4000 /$ techne.438

ISSN : 2534-5168

Éditeur

C2RMF

Édition imprimée

Date de publication : 1 décembre 2018

Pagination : 84-89

ISBN : 978-2-11-152829-1

ISSN : $1254-7867$

Référence électronique

Camille Devilliers et Faisl Bousta, « Les rayons UV-C comme solution alternative aux traitements chimiques pour l'élimination des micro-organismes chlorophylliens », Technè [En ligne], 46 | 2018, mis en ligne le 19 décembre 2019, consulté le 22 juillet 2020. URL : http://journals.openedition.org/techne/ 438 ; DOI : https://doi.org/10.4000/techne.438

\section{(c) $(1) \odot$}

La revue Technè. La science au service de l'histoire de l'art et de la préservation des biens culturels est mise à disposition selon les termes de la Licence Creative Commons Attribution - Pas d'Utilisation Commerciale - Pas de Modification 4.0 International. 


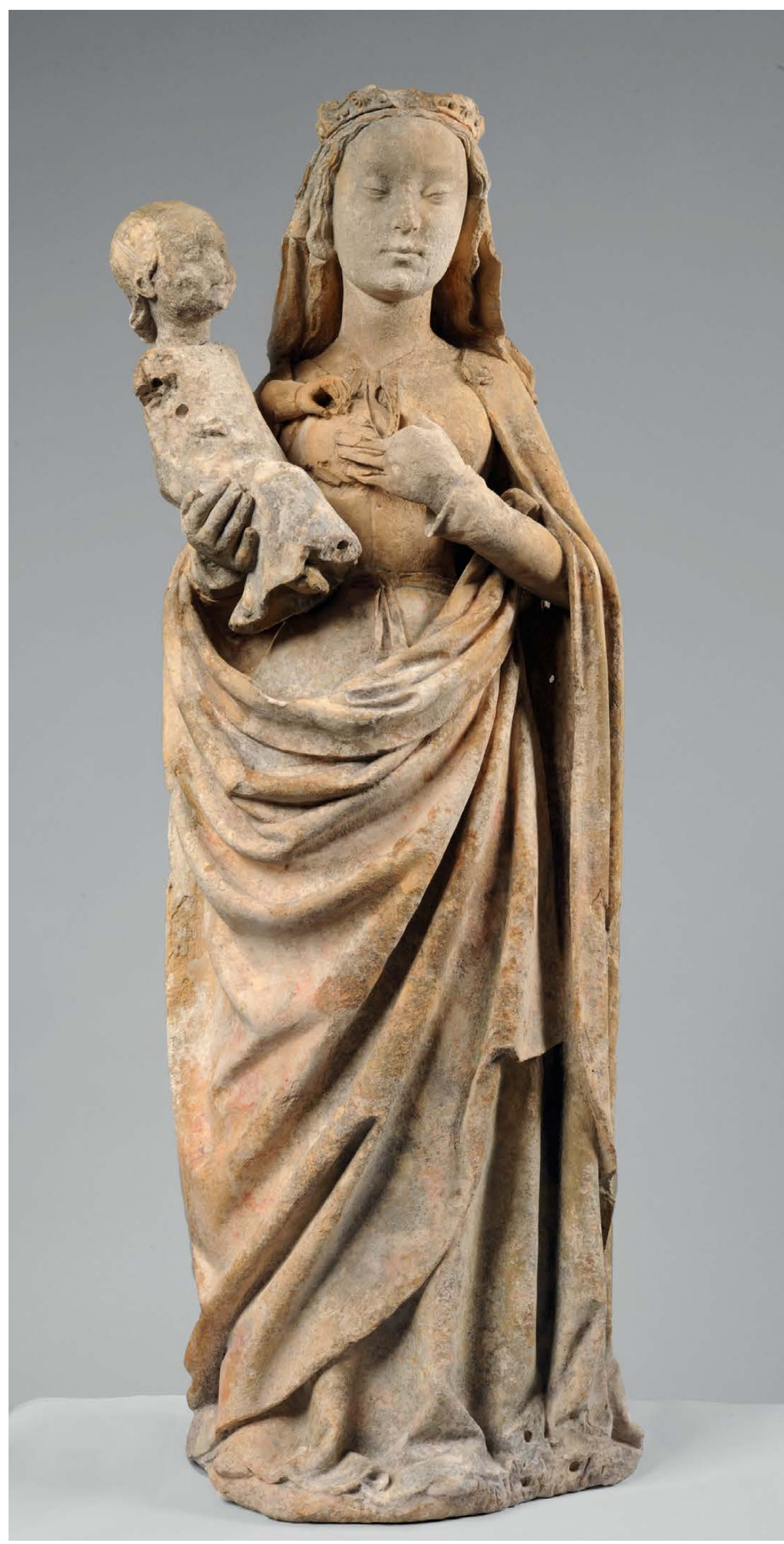

Fig. 1. La Vierge à l'Enfant après les traitements de restauration,

1480-1500, pierre calcaire avec traces de polychromie, musée Sainte-Croix, Poitiers (H. $102 \mathrm{~cm}$ ).

(C) Inp/G. Vanneste. 
Camille Devilliers

Faisl Bousta

Avec la collaboration

de Juliette Lévy

Les rayons UV-C comme solution alternative aux traitements chimiques pour l'élimination des micro-organismes chlorophylliens. Une étude de cas : la Vierge à l'Enfant de Poitiers

The use of UVC rays as an alternative solution to chemical treatments to eliminate chlorophylian microorganisms: the Poitiers Virgin and Child

Résumé. L'étude et la restauration de la sculpture de la Vierge à l'Enfant du musée Sainte-Croix de Poitiers a été l'occasion d'appliquer une méthode innovante de traitement des microorganismes chlorophylliens : l'exposition aux rayons $U V$-C . L'euvre en pierre calcaire, possédant encore des traces de polychromie, avait été conservée en extérieur et était colonisée par les algues, mousses et lichens. La problématique du nettoyage de ces micro-organismes a entraîné une étude technico-scientifique menée au Laboratoire de Recherche des Monuments Historiques. L'exposition sous rayons $U V$-C, nocifs pour les organismes vivants, présentait l'intérêt d'éviter l'utilisation d'un produit biocide.

Les bons résultats de l'étude ont permis d'appliquer cette technique sur la Vierge à l'Enfant, une première dans le domaine de la restauration de sculpture en pierre.

Mots-clés. Pierre calcaire, micro-organismes, rayons $U V-C$, LRMH, Inp, polychromie, Poitiers.
Abstract. The study and restoration of the sculpture of the Virgin and Child in the Musée Sainte-Croix, Poitiers, provided an occasion to apply an innovative method of treatment for chlorophylian microorganisms: exposure to UVC rays. The limestone work, which still bears traces of polychromy, had been kept outside and invaded by algae, moss and lichens. Problems related to cleaning these microorganisms led to a technico-scientific study conducted at the Laboratoire de Recherche des Monuments Historiques (LRMH). Exposure to UVC rays, which have a harmful effect on living organisms, proved of interest because it avoided the use of a biocidal product. The positive results of the study enabled this technique to be applied to the Virgin and Child, a first in the field of restoration of stone sculpture.

Keywords. Limestone, microorganisms, UVC rays, LRMH, INP (Institut national du patrimoine), polychromy, Poitiers.

\section{Introduction}

En 2013, le musée Sainte-Croix de Poitiers a reçu en donation une sculpture exceptionnelle représentant une Vierge à l'enfant (inv. 2013.6.1) (fig. 1). L'œuvre, datée des années 1480-1500, est en pierre calcaire et possède des traces de polychromie. À ce jour, le dépouillement des archives de la famille poitevine à l'origine de la donation et les recherches documentaires n'ont pas permis de déterminer la provenance de l'œuvre.

À son arrivée au musée, son état de conservation était très critique (fig. 2) : le visage de la Vierge s'était fracturé et l'Enfant était brisé en deux fragments. Le séjour de l'œuvre dans un jardin privé pendant un peu plus de cent ans avait provoqué des boursouflures dans la pierre et la perte presque totale de la polychromie ${ }^{1}$. Mais surtout, une importante colonisation biologique s'était développée sur la face de l'œuvre, regroupant micro-algues, mousses et lichens. La problématique de l'élimination de ces micro-organismes a fait l'objet de notre étude technico-scientifique dans le cadre du mémoire de fin d'année du master de restaurateur du patrimoine de l'Institut national du patrimoine ${ }^{2}$.

\section{L'élimination des micro-organismes}

Plusieurs méthodes sont utilisées actuellement pour éliminer les micro-organismes chlorophylliens. Celles-ci impliquent pour la plupart une action mécanique ou l'utilisation de produits chimiques, le plus souvent accompagnées d'un nettoyage et d'un rinçage aqueux. Or l'état de dégradation de la sculpture et la fragilité de la surface ne permettaient pas l'apport d'eau ni une action mécanique de type brossage.

Les méthodes mécaniques, telles que la projection de vapeur d'eau sous faible pression ou le système d'injectionextraction ${ }^{3}$, ont donc été écartées. Seule la technique abrasive du micro-sablage nous intéressait car elle permettait d'effectuer un nettoyage léger et contrôlé, mais elle ne pouvait être

Camille Devilliers, restauratrice de sculpture (camillejeannedevilliers@gmail.com). Faisl Bousta, ingénieur d'études, responsable du pôle Microbiologie au Laboratoire de Recherche des Monuments Historiques (faisl.bousta@culture.gouv.fr). Avec la collaboration de Juliette Lévy, restauratrice de sculpture et responsable de l'atelier sculpture de l'Institut national du patrimoine. 

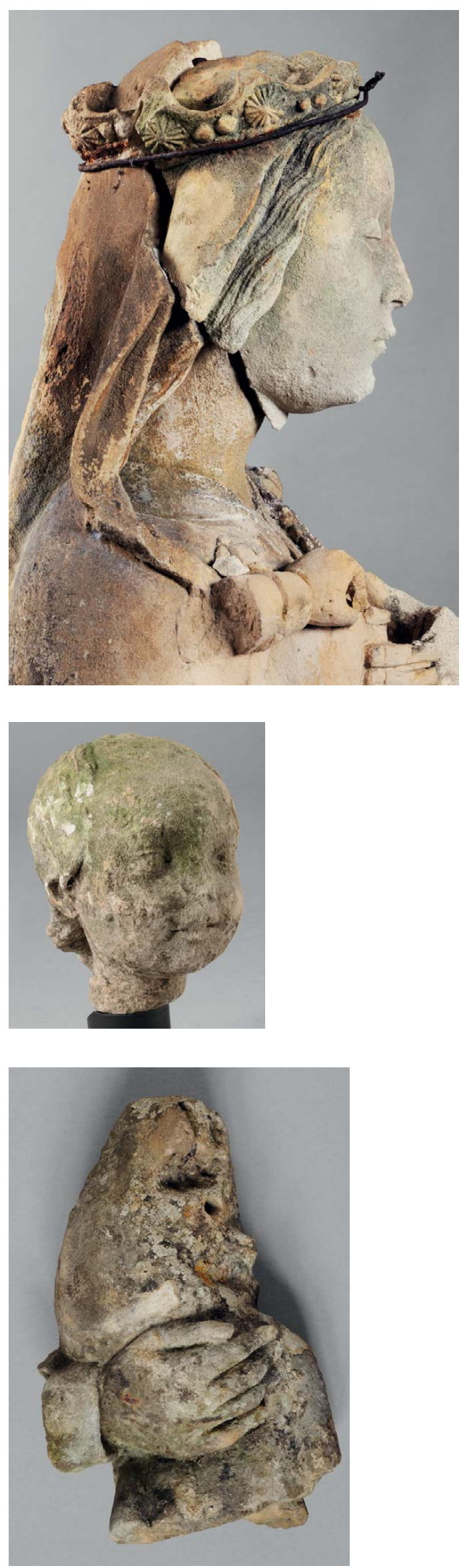

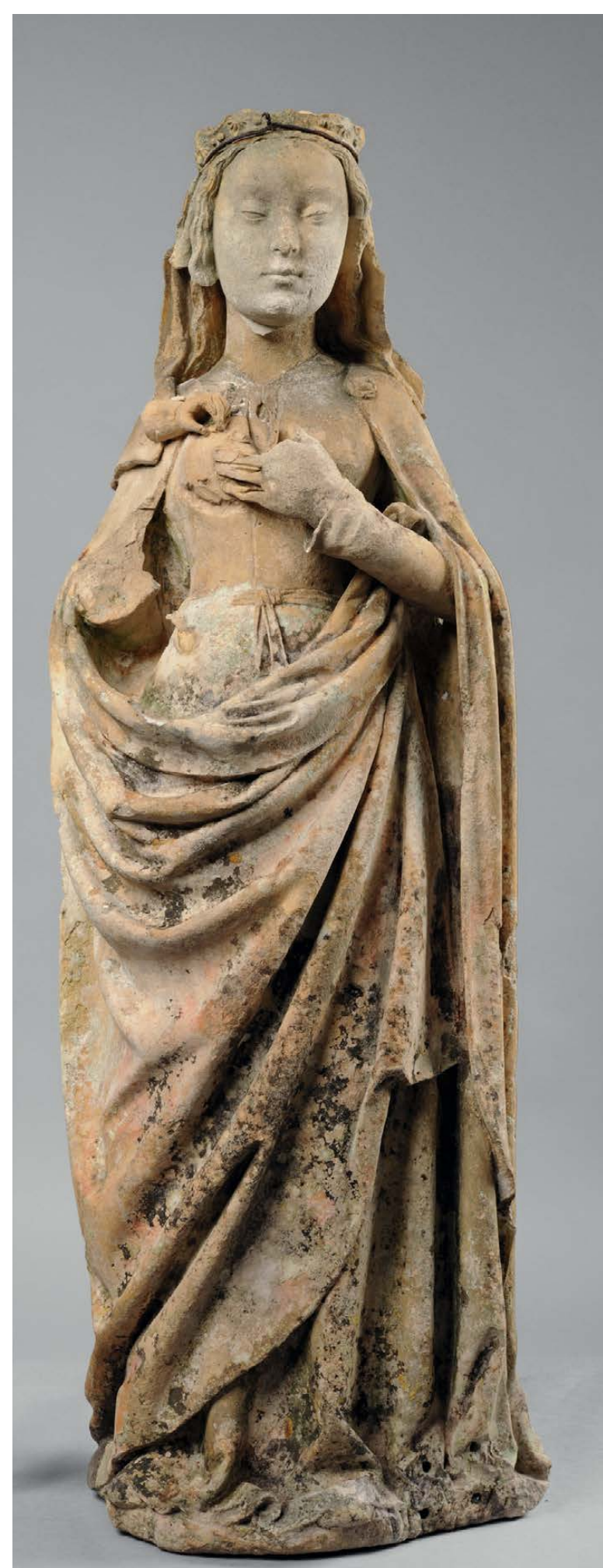

Fig. 2. La Vierge à l'Enfant avant les traitements de restauration. (c) Inp/G. Vanneste. 
efficace que sur une colonisation biologique asséchée (morte).

Parmi les méthodes chimiques, nous avons écarté l'utilisation de gaz (comme l'oxyde d'éthylène), du fait de sa toxicité, ainsi que l'emploi d'un antibiotique ${ }^{4}$. L'application d'un produit biocide reste l'une des méthodes les plus utilisées actuellement. Cependant, ces algicides à base de sels d'ammonium quaternaire nécessitent un rinçage à l'eau accompagné d'un brossage doux pour éliminer les micro-organismes morts : ils ne pouvaient donc être appliqués sur la sculpture.

Enfin, les méthodes physiques comme les rayons ionisants et non ionisants, tels que les rayons gamma ou laser, impliquaient l'utilisation de machines puissantes, donc coûteuses et peu accessibles. Ces contraintes ne convenaient pas à notre étude pour laquelle nous cherchions une méthode facile à mettre en œuvre pour les restaurateurs.

\section{Les rayons UV-C}

L'exposition aux rayons UV-C est une méthode déjà utilisée aujourd'hui, pour la stérilisation de l'air conditionné par exemple. Récemment, cette méthode a été appliquée dans la grotte des Moidons (Jura), dans le cadre d'une thèse de Fabien Borderie intitulée Utilisation du rayonnement $U V-C$ comme méthode alternative aux produits chimiques dans la lutte et le contrôle de la prolifération des micro-organismes sur les matériaux du patrimoine. Cette thèse, réalisée au LRMH en collaboration avec le Laboratoire Chrono-Environnement de la faculté des sciences de Besançon, a constitué la base de notre recherche.

Les rayons UV-C se situent dans le spectre électromagnétique entre la lumière visible et les rayons $\mathrm{X}$ et leur longueur d'onde se situe entre 200 et $280 \mathrm{~nm}$. Ils sont hautement énergétiques et donc nocifs pour les organismes vivants sur lesquels ils ont une action germicide. En effet, ces rayons causent des dommages irréversibles au niveau cellulaire, avec notamment la dégradation des membranes et la décoloration des cellules par destruction des pigments chlorophylliens et carotènes et, au niveau moléculaire, avec la fragmentation de l'ADN et la dégradation des protéines.

Suite à la destruction des pigments chlorophylliens, la colonisation biologique va visuellement s'éclaircir et blanchir progressivement.

Les rayons UV-C, émis par des tubes néons, ne pénètrent pas en profondeur dans la pierre. L'efficacité du traitement dépend de plusieurs paramètres : l'épaisseur du biofilm, l'énergie appliquée, la distance de la source lumineuse par rapport au biofilm et la durée d'exposition. Un temps de repos est nécessaire pour constater une pleine efficacité du traitement.

L'utilisation des rayons UV-C présentait ici les avantages de n'introduire aucun produit, et de n'entraîner aucune action mécanique. De plus, le matériel nécessaire était accessible et la mise en œuvre assez simple. La question s'est donc posée de savoir si l'exposition aux rayonnements UV-C serait aussi efficace qu'une méthode habituellement utilisée, telle que le traitement biocide, sur une sculpture. Un protocole a donc été mis au point et des essais ont été réalisés au Laboratoire de Recherche des Monuments Historiques (LRMH).

\section{Essais en laboratoire}

\section{La collecte des échantillons}

L'analyse $^{5}$ de la pierre calcaire de la Vierge à l'Enfant a permis de rapprocher celle-ci de la pierre extraite des carrières de Migné-Auxances situées au nord-ouest de Poitiers. Certains blocs de pierre, restés en extérieur depuis une centaine d'années, présentaient une colonisation biologique similaire à celle de notre sculpture. Après quelques coups de massue, des échantillons d'une quinzaine de centimètres ont pu être prélevés par nos soins.

\section{L'élimination des micro-organismes}

Pour comparer l'efficacité des rayons UV-C et des biocides, le produit chimique Biotin $\mathrm{T}^{\circledR}$, recommandé par le LRMH, a été appliqué sur un premier lot d'échantillons.

Les échantillons traités avec les rayons UV-C ont été placés sous une boîte en bois conçue par le laboratoire (60 x 34 x $22 \mathrm{~cm}$ ) et équipée de trois tubes néons germicides G15T8 Philips ${ }^{\circledR}$. La longueur d'onde maximale émise par ces tubes néons ${ }^{7}$ est de 253,7 $\mathrm{nm}$ et leur puissance combinée est de 45 watts. Du papier d'aluminium tapissant la boîte permettait d'augmenter la réflexion des rayons UV-C et de concentrer un maximum d'énergie à la surface des échantillons. La boîte ne devait laisser filtrer aucun rayon vers l'extérieur, ces derniers étant nocifs pour l'homme.

Ce lot a été traité en deux temps :

$-1^{\text {re }}$ exposition de 19 heures, suivie d'un nettoyage de la surface par un brossage doux à l'eau ${ }^{8}$. Une fois les échantillons secs, le niveau d'élimination des micro-organismes n'étant pas satisfaisant, l'exposition a été renouvelée.

$-2^{\mathrm{e}}$ exposition de 18 heures 30, suivie à nouveau d'un nettoyage de la surface des échantillons.

Lorsque les échantillons ont été sortis de la boîte, un éclaircissement des surfaces a été constaté instantanément ainsi qu'un léger échauffement de la pierre.

\section{Résultats}

Les résultats des tests ont été évalués de différentes manières : l'observation visuelle et les mesures colorimétriques ont permis de constater que les deux traitements avaient été efficaces et que les mousses et les algues avaient été éliminées (fig. 3).

Cependant, aucun des deux traitements n'a eu d'effets sur les lichens épais et incrustés dans la pierre, surtout les lichens noirs. En revanche, ils étaient devenus secs et cassants, et ont pu être facilement éliminés de manière mécanique (dégagement au scalpel) sans altérer l'épiderme de la pierre. 


\section{Application sur la sculpture}

Suite aux résultats de l'étude technico-scientifique, l'application du traitement sur l'œuvre a été approuvée par le responsable de l'œuvre, Pascal Faracci, directeur des musées de Poitiers.

\section{Conception de la boîte}

À l'image de celle conçue par le LRMH, la boîte a été équipée de neuf tubes néons germicides de la marque Philips ${ }^{\circledR}$, d'une puissance de 10 watts chacun ${ }^{9}$. Leur répartition homogène permettait aux rayons d'atteindre toutes les faces de l'ouvre et la sculpture a été pivotée sur elle-même pendant le traitement afin que les rayons UV-C atteignent les zones très profondément creusées (fig. 4). L'intérieur de la boîte a également été tapissé de feuilles d'aluminium. Afin de contrôler l'allumage des tubes néons et la fonctionnalité de notre circuit électrique, un capteur d'UV-C-mètre a été posé sur la main gauche de la Vierge. La composition de la polychromie et du liant étant encore inconnue au moment du traitement, ces zones ont été protégées par du papier aluminium à titre préventif.

\section{Mise en cuvre du traitement}

Lors de l'étude technico-scientifique, nous avions constaté un léger échauffement des échantillons après traitement. Afin de surveiller l'évolution de la température dans la boîte, un capteur thermique a été placé sur la sculpture. Les mesures ont indiqué que la température augmentait régulièrement de $2{ }^{\circ} \mathrm{C}$ par heure : la pierre ne subissait pas un choc thermique mais un échauffement progressif. Suite à ces résultats, il a été décidé de traiter la sculpture en alternant une phase de traitement (la nuit, durant 15 heures) et une phase de repos (le jour). La température augmenterait ainsi de $30{ }^{\circ} \mathrm{C}$ et atteindrait une température de $50^{\circ} \mathrm{C}$ dans la boîte, jugée acceptable pour la pierre calcaire ${ }^{10}$. L'alternance entre les phases a ainsi évité une surchauffe du matériau et nous a permis de réaliser d'autres interventions en parallèle. La durée d'exposition a été choisie en fonction du résultat visuel, c'est-à-dire en fonction de la disparition des algues et du jaunissement des mousses : au total, la sculpture a été exposée environ 60 heures. Les fragments de l'Enfant et le visage de la Vierge ont été traités dans la boîte du LRMH. En effet, aucun effet visuel n'ayant été constaté au bout de 30 heures d'exposition dans la grande boîte, la boîte de plus petite dimension a été utilisée pour réduire la distance d'exposition.

\section{Résultats}

À l'issue du traitement, les algues vertes ont complètement disparu (fig. 5). Nous n'avons observé aucune modification visuelle de la polychromie suite à l'échauffement de la pierre. Seuls les lichens sont restés à la surface de l'œuvre. Ils ont été éliminés à l'aide d'un scalpel à lame émoussée, afin d'éviter de rayer la surface de la pierre. Les lichens noirs plus

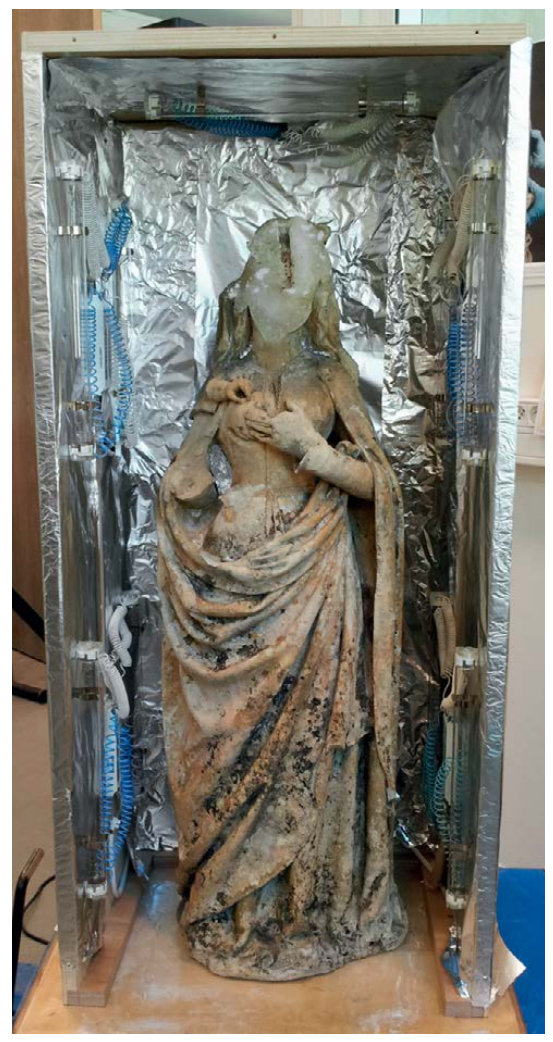

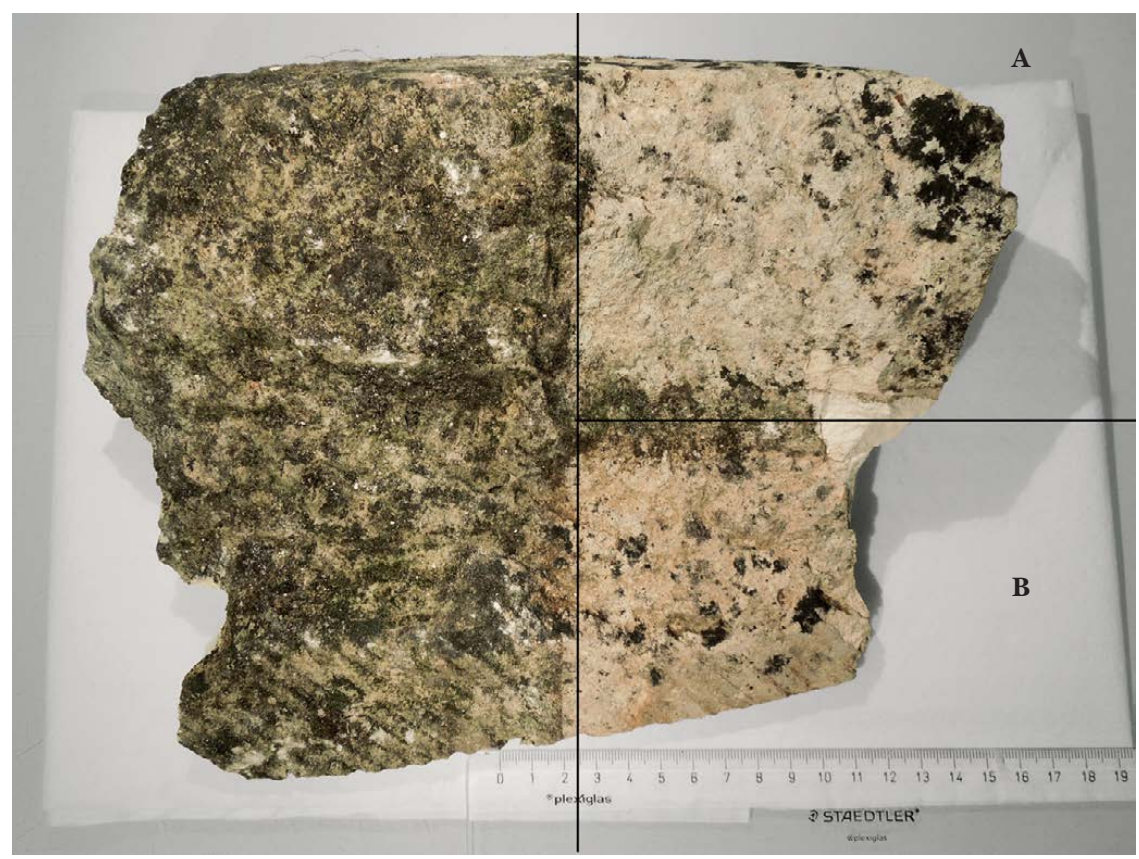

Fig. 3. Échantillon de carrière $(23,5 \times 25,3 \times 8,5 \mathrm{~cm})$ avec la zone à gauche avant traitement et la zone à droite après traitement. Dans la zone après traitement, en haut : A. Traitement sous rayons UV-C, en bas : B. Traitement biocide. () Inp/C. Devilliers.

Fig. 4. La sculpture placée dans la boîte de traitement. (C) Inp/C. Devilliers. 
incrustés ont été en partie éliminés par micro-sablage : après des tests sur les échantillons du protocole, la poudre d'oxyde d'alumine à $29 \mu \mathrm{m}$ a été retenue ${ }^{11}$.

\section{Conclusion}

L'étude technico-scientifique menée autour de la Vierge à l'Enfant a été l'occasion d'appliquer avec succès les rayons UV-C pour la première fois sur une œuvre sculptée en pierre.

L'influence des rayons sur la polychromie n'a pas été étudiée au vu des très petites quantités préservées sur l'œuvre ${ }^{12}$. Avant d'envisager l'application des rayons UV-C sur des sculptures en pierre polychromées, d'autres recherches devront être menées afin de déterminer leurs effets sur les matériaux colorés.

La diffusion des pigments noirs contenus dans les lichens a laissé des traces sur la pierre. Cette étude a aussi été l'occasion de réaliser des tests préliminaires avec la lumière pulsée (émission de flash de lumière blanche intense) pour l'élimination de ces pigments noirs. Les premiers résultats sont prometteurs, mais une étude complète devrait permettre de déterminer des paramètres d'application plus précis (énergie appliquée, temps d'exposition et épaisseur du biofilm).

\section{Remerciements}

Nous remercions Pascal Faracci, directeur des musées de Poitiers, Béatrice de Chancel-Bardelot, conservateur du patrimoine au musée de Cluny-musée national du Moyen Âge, et Lise Leroux, ingénieure de recherche au pôle Pierre du LRMH.

\section{Notes}

1. Seuls quelques îlots de bleu et d'or sont préservés dans la chevelure et les vêtements de la Vierge.

2. Mémoire encadré par J. Lévy, F. Bousta et B. de Chancel-Bardelot, avec la collaboration de P. Faracci.

3. Le fonctionnement du système d'injection-extraction est le suivant : une machine, munie d'un bras et d'un embout, projette et aspire alternativement de l'eau.

4. Les bactéries peuvent développer une résistance à l'antibiotique et réduire l'efficacité du traitement.

5. Analyse réalisée par Lise Leroux, ingénieure au pôle Pierre, LRMH.

6. Le Biotin $\mathrm{T}^{\circledR}$ est constitué notamment d'un sel d'ammonium quaternaire et est utilisé dilué à $3 \%$ dans l'eau.

7. La manipulation des tubes doit se faire avec des gants de protection afin de protéger la peau de l'arc de mercure générateur d'ultraviolets présents dans les tubes.

8. Le recouvrement biologique étant plus dense que celui de la sculpture, nous avons décidé de brosser les micro-

organismes après le premier traitement pour faciliter la pénétration des rayons.
9. Fournisseur: France Lampes ${ }^{\circledR}$.

10. L'échauffement du matériau peut être évité par la mise en place d'une ventilation dans la boîte (elle n'a pas été mise en place ici par manque de temps durant l'étude).

11. Pression de 0,5 bar pour un débit de poudre de 2 , avec une buse de $0,8 \mathrm{~mm}$ de diamètre.

12. Les analyses, réalisées par le laboratoire scientifique de l'Inp, ont révélé la présence de bleu azurite, d'indigo, d'or, d'une préparation au blanc de plomb et de pigments au plomb.

\section{Bibliographie}

Delgado Rodriguez J., Valero J., 2003, "A brief note on the elimination of dark stains of biological origin”, Studies in Conservation, IIC, $\mathrm{n}^{\circ} 48$, p. 17-22.

Orial G., Roquebert M.-F., Bousta F. et al., 2005, « Les altérations biologiques et les biens patrimoniaux », Monumental, 2005-1, p. 94-117.

\section{Documents inédits}

Borderie F., 2014, Utilisation du rayonnement $U V$-C comme méthode alternative aux produits chimiques dans la lutte et le contrôle de la prolifération des microorganismes sur les matériaux du patrimoine, université de Franche-Comté, UFR Sciences et Techniques, thèse soutenue le 14 novembre 2014, 312 p.

Kapp J., 2007, Étude et restauration de trois sculptures du Château-Musée de Dieppe : Saint Jacques en plâtre d'Eugène Paul Bénet (vers 1898), Tête de saint Jacques et Livre, fragments en pierre de Caen ( fin $X V^{e} /$ début $X V I^{e}$ siècle), Tours : École supérieure des Beaux-Arts, cycle conservation-restauration des œuvres sculptées, 197 p.

Mauny P., 2004, Étude d'un buste de marbre du XVII siècle représentant Rome du musée national du château de Versailles:

Traitement des altérations liées à son exposition en extérieur et recherche appliquée sur l'adaptation de la résistance mécanique du goujonnage, Inp, département des restaurateurs du patrimoine, $93 \mathrm{p}$. 